\title{
BCG Infection due to MPT64-Negative Strain: A Diagnostic Challenge
}

\author{
Parasmal Suresh, ${ }^{1}$ Lalitha Biswas, ${ }^{1}$ Vinitha Prasad, ${ }^{2}$ Anil Kumar, ${ }^{3 \star}$ Suchitra Sivadas, ${ }^{2}$ Sadia Khan, ${ }^{3}$ and Raja Biswas ${ }^{1}$ \\ ${ }^{1}$ Centre for Nanosciences and Molecular Medicine, Amrita Institute of Medical Sciences and Research Centre, Amrita VishwaVidyapeetham, \\ Kochi, India; ${ }^{2}$ Department of Paediatrics, Amrita Institute of Medical Sciences and Research Centre, Kochi, India; ${ }^{3}$ Department of Microbiology, \\ Amrita Institute of Medical Sciences and Research Centre, Kochi, India
}

\begin{abstract}
MPT64 is a 24-kDa immunogenic protein that is widely used as a diagnostic marker for the differentiation of Mycobacterium tuberculosis complex (MTBC) from nontuberculous Mycobacterium (NTM). Unlike Mycobacterium bovis, Bacillus Calmette-Guerin (BCG) vaccine strains with RD2 deletion do not secrete MPT64. Culture isolates from infections due to these strains may be falsely identified as nontuberculous Mycobacterium in the absence of clinical correlation. Here, we present one case each of BCG adenitis and osteitis, both of which were considered as MPT64 card-negative Mycobacterium spp. (i.e., NTM) and were later identified as $M$. bovis BCG Danish 1331 strain. The first case was a 4-month-old female infant admitted with swollen lymph nodes in the left supraclavicular and the left axillary region of 1 month duration. The second case was of a 1-year-and-5-month-old male child who presented with a limp on the left leg and soft tissue swelling of 1 month duration on the anterolateral aspect of the left knee joint. In both cases, BCG vaccine was administered at birth on the left deltoid region and had healed without any complication. Clinical samples in both cases were positive by Xpert tuberculosis/ RIF for MTBC, and cultures grew acid-fast bacilli which were negative by MPT64 assay. The clinical implication of infections due to $M$. bovis BCG is immense as they are inherently resistant to pyrazinamide, and the presence of disseminated BCG infection in young children is a hallmark of serious immune deficiency which needs to be ruled out.
\end{abstract}

\section{INTRODUCTION}

Live attenuated BCG vaccine was introduced to the world in $1921 .{ }^{1}$ In countries like India, with a high burden of tuberculosis (TB), the WHO recommends BCG vaccination of all children at birth to prevent tuberculous meningitis and disseminated TB in young children and infants. ${ }^{1}$ Among the various BCG strains, Glaxo 1077, Pasteur 1173, Danish 1331, and Tokyo 172 account for almost $90 \%$ of the vaccines worldwide. $^{1,2}$ Whereas Pasteur 1173 and Danish 1331 are considered as strong strains (higher vaccine efficacy), Glaxo 1077 and Tokyo 172 are weak strains (lower vaccine efficacy). ${ }^{1,2}$ The genealogy of BCG vaccine strains based on historical data shows that BCG strains differ in the presence of mpb64 gene and the number of copies of IS6110 gene.,4 The ancestral BCG strain obtained before 1925 had two copies of IS6110 and mpb64 genes, but subsequently loss of one IS6110 happened in 1925-1926 followed by loss of mpb64 gene around 1927-1931. ${ }^{3,4}$ The mpb64 gene is present in BCG-Japan, BCG-Sweden, BCG-Moreau, BCGBirkhaug, and BCG-Russia, whereas it is absent in BCG-Danish, BCG-Pasteur, BCG-Glaxo, BCG-Tice, and BCG-Connaught. Complications due to $B C G$ vaccination are influenced by $B C G$ strain, dose, age, technique of immunization, and the underlying immune status of the vaccines. ${ }^{2,3}$ The incidence of severe complications ranges from 2 to 1,000 per million vaccinations and is most often seen in patients with primary immunodeficiency disorders such as chronic granulomatous disease, severe combined immunodeficiency, type 1 cytokine axis defect, DiGeorge syndrome, and HIV infection. ${ }^{1,3}$ Severe complications may require chemotherapy with antiTB drugs, which get complicated by the inherent resistance to pyrazinamide in all Mycobacterium bovis strains. ${ }^{1,5}$ Because of inherent resistance to pyrazinamide, the $\mathrm{CDC}$ recommends a triple-drug regimen consisting of rifampicin, isoniazid, and

\footnotetext{
* Address correspondence to Anil Kumar, Department of Microbiology, Amrita Institute of Medical Sciences and Research Centre, Amrita VishwaVidyapeetham, Ponekkara, Kochi 682041, India. E-mail: vanilkumar@aims.amrita.edu
}

ethambutol with treatment duration being extended to 9 months for treatment of disease due to $M$. bovis. ${ }^{6}$ Disseminated BCG infection in young children could be an indication of serious immune deficiency, which needs to be investigated and ruled out. Therefore, differentiation of $M$. bovis BCG from Mycobacterium tuberculosis as well as from nontuberculous mycobacteria (NTM) is crucial for appropriate treatment. Assays for MPT64 protein have been recommended by the Foundation for Innovative New Diagnostics for rapid identification of the M. tuberculosis complex using the BACTEC MGIT 960 liquid culture system (Becton Dickinson Diagnostics, Sparks, MD). ${ }^{7}$ The assays are routinely performed all over the world, both in developed and developing countries, as suggested by two meta-analyses on their performance in accurately identifying the $M$. tuberculosis complex in liquid culture. $^{8,9}$ We report two cases of BCG infection due to $M$. bovis BCG strains which were MPT64 negative.

\section{SHORT REPORT}

Case 1. A 4-month-old female infant was admitted with complaints of high-grade intermittent fever, cough, and difficulty breathing. A swelling in the neck and the axillary region was noticed 3 months earlier. She was also diagnosed to have asplenia and patent ductus arteriosus at the age of 3 months. Clinical examination revealed bilateral inguinal hernia, coarse facies, and matted lymph nodes in the left axilla and the supraclavicular region. BCG vaccine administered on day 2 of life on the left deltoid region had healed without any complications. Chest $\mathrm{X}$-ray showed clear lung fields without any hilar nodes or mediastinal widening.

Case 2. A 1-year-and-5-month-old male child, developmentally normal for age, presented with a limp on the left leg and soft tissue swelling of 1 month duration on the anterolateral aspect of the left knee joint. The size of the swelling progressively increased over 2 weeks from about $1 \times 1 \mathrm{~cm}$ to $2 \times 2 \mathrm{~cm}$. There were no complaints of associated fever, pain over swelling, skin changes, or weight loss. The child was immunocompetent with no underlying disorders, and the BCG 
vaccine administered on the left deltoid region at birth healed without any complication.

Written informed consent was obtained from the legal guardians of both the patients. Pus aspirated from the left supraclavicular lymph node in the first case and tissue from the excised swelling in the second case were subjected to acid-fast stain, aerobic cultures, Mycobacterium tuberculosis complex (MTBC) PCR using Xpert ${ }^{\circledR}$ MTB/RIF assay (Cepheid Inc., Sunnyvale, CA), and liquid mycobacterial cultures using BACTEC MGIT 960 (Becton Dickinson Diagnostics, Sparks, MD). The clinical mycobacterial isolates were compared with $M$. tuberculosis $\mathrm{H} 37 \mathrm{Ra}$, MPT64-positive M. bovis BCG-Russia (Moscow 368, Serum Institute of India), and MPT64-negative M. bovis BCG-Denmark (Danish 1331, Green Signal Bio Pharma Pvt Ltd. Chennai, India) strains because in India both these strains are used for vaccination. Genomic DNA was isolated from the cultures of clinical isolates, vaccine strains, and $M$. tuberculosis H37Ra. To differentiate between $M$. bovis and the other MTBC complex organisms, gyrB gene was amplified by PCR and the 1,020-bp PCR product was subjected to restriction fragment length polymorphism (RFLP) using Taql and sequencing. The sequences obtained were analyzed using the BLASTN algorithm. To detect and differentiate $M$. bovis BCG from $M$. tuberculosis and $M$. bovis, PCR amplifications of the RD1 and RD4 regions were performed using the primers, as described earlier (Table 1). ${ }^{10}$

Smear for acid-fast bacilli (AFB) and aerobic cultures of the pus and tissue were negative. Mycobacterial cultures grew AFB showing cord formation. PCR of pus and tissue using Xpert MTB/RIF was positive for MTBC with rifampicin resistance not detected, indicating that both the specimens belong to MTBC. But both the MGIT culture isolates were negative for the MPT64 protein immunochromatographic test (ICT) (Standard Diagnostics, Seoul,. South Korea), suggestive of NTM. To resolve these conflicting results and identify the species of these two MPT64 card-negative and Xpert MTB/ RIF-positive MGIT culture isolates, PCR amplification, RFLP, and sequencing methods were carried out.

RFLP patterns of the clinical isolates were identical to those of the vaccine strains using Taql (Figure $1 \mathrm{~A}$ and B). The RD1 PCR was used to differentiate $M$. bovis BCG (196 bp) from M. tuberculosis/M. bovis (146 bp). The RD4 PCR was used to differentiate between $M$. tuberculosis (172 bp) and $M$. bovis/ M. bovis BCG (268 bp) (Figure 1E and F). RD4 PCR results identified the culture isolates as M. bovis/M. bovis BCG (268 bp), and RD1 PCR identified them as $M$. bovis BCG. Sequencing of the gyrB and the RD4 PCR products identified the clinical isolates as $M$. bovis BCG Danish 1331 (Figure 1B and C).

Based on these observations, the first patient underwent aspiration of the supraclavicular lymph node and biopsy of the left axillary lymph node, whereas the second patient underwent excision of the soft tissue swelling and treatment with modified first-line antitubercular drugs with replacement of pyrazinamide with streptomycin. At 1-year follow-up, the first child is doing well with complete resolution of lymphadenitis without any relapse. The second child completed 6 months of antitubercular therapy with complete resolution of symptoms.

\section{DISCUSSION}

The identification of $M$. bovis BCG is not straightforward as it belongs to MTBC, a group of highly related organisms comprising M. tuberculosis, Mycobacterium africanum, M. bovis, Mycobacterium microti, and Mycobacterium canettii. ${ }^{11}$ The viable and actively dividing cells of the members of MTBC secrete a $24-\mathrm{kDa}$ secretary protein which remains stable in the culture medium for at least 1 year., ${ }^{2,7}$ The introduction of rapid ICT for the detection of MPT64 protein has made the differentiation of MTBC from NTM very simple and economical. ${ }^{8}$ However, M. bovis BCG strains with deletion or mutation in the mpb64 gene cannot be detected with this assay. ${ }^{4}$ Single-nucleotide polymorphisms that exist in the gyrB gene of the MTBC were used to differentiate $M$. bovis from the other MTBC species by performing RFLP using the restriction enzyme Taql. ${ }^{12}$ Previous studies have shown the absence of regions of difference $(\mathrm{RD}) \mathrm{RD} 1, \mathrm{RD}$, and $\mathrm{RD} 10$ in all $B C G$ vaccine strains and the presence in TB strains, although $M$. bovis lacks the RD4 region. Non-BCG $M$. bovis is distinguished from BCG by the presence of RD1 and the absence of RD9. ${ }^{10}$ In this study, we have used the RD1 region- and RD4 region-specific amplifications to differentiate between $M$. tuberculosis, $M$. bovis, and $M$. bovis BCG.

The diagnostic algorithm for pediatric TB in India advocates the upfront use of cartridge-based nucleic acid testing (Xpert MTB/RIF) and cultures, if available. ${ }^{13}$ Both these tests will not differentiate $M$. tuberculosis from $M$. bovis and may lead to inappropriate treatment with pyrazinamide, to which $M$. bovis is inherently resistant. Moreover, the use of MPT64-negative BCG strain will lead to misidentification as NTM in mycobacterial cultures. Pediatric infections due to NTM are very rare and may occur in children with underlying immunodeficiency. A study from India reported that $75 \%$ of infants with BCG-related disease have regional or localized involvement, whereas only $25 \%$ had disseminated disease. ${ }^{14}$ Among patients with disseminated disease, only one patient had IFNY-R1 gene mutation. ${ }^{14}$ The use of two different strains for vaccination in the same population, one with and the other without the mpb64 gene, further complicates the problem of identification. In India, the public sector uses MPT64-negative

TABLE 1

List of tests performed to examine the specimens and culture isolates

\begin{tabular}{lll}
\hline \multicolumn{1}{c}{ Method } & \multicolumn{1}{c}{ Test performed } & \multicolumn{1}{c}{ Result } \\
\hline Microbiological identification (clinical specimen) & Acid-fast staining & Negative \\
& BACTEC MGIT liquid mycobacterial culturing & Positive \\
Rapid Methods (PCR/immunochromatographic) & Xpert MTB/RIF (clinical specimen) & Positive for MTBC/RIF sensitive \\
& SD Bioline MPT64 card assay (culture isolates) & Negative for MTBC \\
Molecular biological identification (culture & gyrB PCR-RFLP using Taql & M. bovis \\
isolates) & RD1 & M. bovis BCG \\
& RD4 & M. bovis/M. bovis BCG \\
& DNA sequencing gyrB gene & M. bovis BCG Danish 1331 \\
\hline
\end{tabular}


A

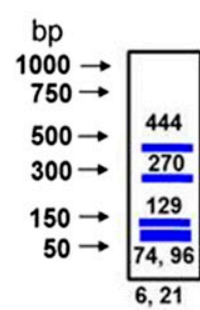

\section{C} M_africanum M_canetti Mbovis M_bovis_BCG $\overline{M t b}$ Hз $\bar{R} A$ M.microti
GCGCCGACCAAGAAGACGGGGTCAACGGTGCGGTTCTGGGCCGACCCCGC GCGCCGACTAAGAAGACGGGGTCAACGGTGCGGTTCTGGGCCGACCCCGC GCGCCGACCAAGAAGACGGGGTCAACGGTACGGTTCTGGGCCGACCCCGC GCGCCGACCAAGAAGACGGGGTCAACGGTACGGTTCTGGGCCGACCCCGC GCGCCGACCAAGAAGACGGGGTCAACGGTGCGGTTCTGGGCCGACCCCGC GCGCCGACCAAGAAGACGGGGTCAACGGTGCGGTTCTGGGCCGACCCCGC

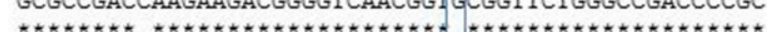

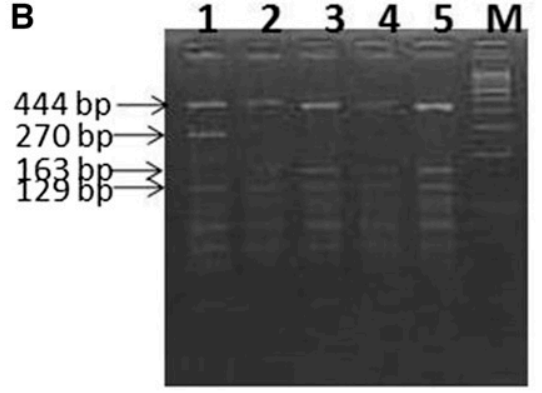

D。

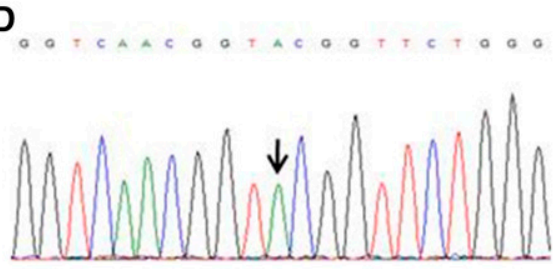

E

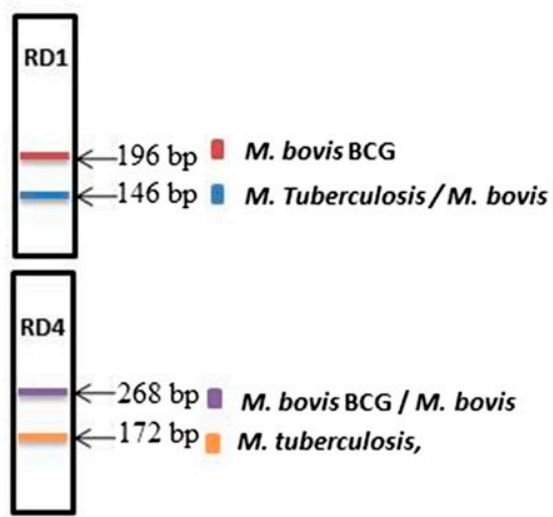

$\mathbf{F}$

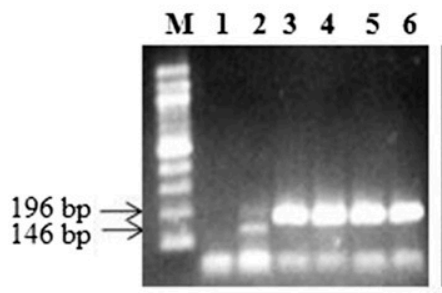

RD4 PCR

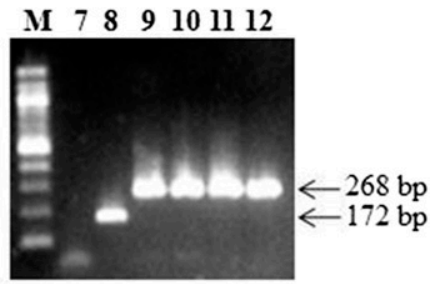

FIGURE 1. (A) Schematic representation of the Taql restriction pattern of the 1,020-bp gyrB fragment among the members of the MTBC. (B) RFLP pattern obtained after the digestion of the 1,020-bp gyrB PCR product with the Taql restriction enzyme. Lane 1: Mycobacterium tuberculosis H37Ra, 2: Mycobacterium bovis BCG-Russia (Moscow 368) (Serum Institute of India), 3: M. bovis BCG-Denmark (Danish 1331) (Green Signal Biopharma, India), 4 and 5: MGIT culture-positive and MPT64-negative samples, Lane M: 100-bp DNA ladder. A 1020-bp PCR-amplified product was seen in both clinical isolates. (C) Multiple sequence alignment of the discriminatory regions in the gyrB gene between $M$. bovis and $M$. bovis BCG and other members of the MTBC. The black box indicates the single nucleotide variation $\mathrm{G}$ to $\mathrm{A}$. (D) Electropherogram of the gyrB PCR product of the clinical isolate. The arrow indicates the single-nucleotide variation $G$ to A representing $M$. bovis. (E) Schematic representation of RD1 and RD4 PCR amplification band sizes for differentially identifying $M$. bovis BCG and $M$. tuberculosis and $M$. bovis. (F) Agarose gel electrophoresis of RD1 and RD4 PCR products: Lanes 1 to 6 are RD1 PCR products; Lane M: 100-bp DNA ladder, Lane 1: no template control (NTC), Lane 2: M. tuberculosis H37Ra, Lane 3: M. bovis BCG-Russia (Moscow 368), Lane 4: M. bovis BCG-Denmark (Danish 1331), and Lanes 5 and 6: MGIT culture-positive and MPT64-negative samples; Lanes 7 to 12 are RD4 PCR products; Lane 7: NTC, Lane 8: M. tuberculosis H37Ra, Lane 9: M. bovis BCG-Russia (Moscow 368), and Lane 10: M. bovis BCG-Denmark (Danish 1331); and Lanes 11 and 12: MGIT culture-positive and MPT64-negative samples. This figure appears in color at www.ajtmh.org.

Danish 1331 strain which is provided free of cost, whereas the private sector uses the MPT64-positive Russian (Moscow) 368 strain and needs to be bought by the patients. ${ }^{15}$ The knowledge of various strains used for vaccination plays a crucial role in avoiding misidentification in cases of BCGassociated complications. In our case, although the patient was asplenic, she was immunocompetent, and the BCG vaccine lesion healed normally with a scar. The children were vaccinated with Danish 1331 strain; therefore, the culture isolates were negative for the MPT64 antigen. The pus and tissue samples were positive for MTBC using Xpert MTB/RIF as it targets the $r p o B$ gene for identification. However, Xpert MTB/RIF does not differentiate between the members of MTBC, making it not possible to identify $M$. bovis BCG. Because $M$. bovis is inherently resistant to pyrazinamide, accurate identification is necessary for appropriate chemotherapy.
Our case emphasizes the importance of careful use of the MPT64 kit with clinical correlation and combining morphological characteristics or nucleic acid amplification tests to identify MPT64-negative $M$. bovis BCG clinical isolates. Clinicians should also be aware that finding disseminated BCG infection in young children is a hallmark of serious immune deficiency which would need investigation and management.

Received November 15, 2019. Accepted for publication May 6, 2020. Published online June 16, 2020.

Acknowledgment: The American Society of Tropical Medicine and Hygiene (ASTMH) assisted with publication expenses.

Authors' addresses: Parasmal Suresh, Raja Biswas and Lalitha Biswas, Center for Nanosciences and Molecular Medicine, Amrita 
Institute of Medical Sciences and Research Centre, Amrita Vishwa Vidyapeetham, Kochi, India, E-mails: parasmals@aims.amrita.edu, rajabiswas@aims.amrita.edu and biswaslalitha@gmail.com. Vinitha Prasad and Suchitra Sivadas, Department of Paediatrics, Amrita Institute of Medical Sciences and Research Centre, Amrita Vishwa Vidyapeetham, Kochi, India, E-mails: vinithaprasad@aims.amrita. edu and suchitra@aims.amrita.edu. Anil kumar, and Sadia Khan, Department of Microbiology, Amrita Institute of Medical Sciences and Research Centre, Kochi, India, E-mails: vanilkumar@aims.amrita. edu, and sadiakhan20004@aims.amrita.edu.

\section{REFERENCES}

1. World Health Organization, 2004. BCG vaccine. WHO position paper. Wkly Epidemiol Rec 79: 27-38.

2. Davids V et al., 2006. The effect of bacille Calmette-Guerin vaccine strain and route of administration on induced immune responses in vaccinated infants. $J$ Infect Dis 193: 531-536.

3. Behr MA, 2002. BCG—different strains, different vaccines? Lancet Infect Dis 2: 86-92.

4. Harboe M, Nagai S, Patarroyo E, Torres ML, Ramirez C, Cruz N, 1986. Properties of proteins MPB64, MPB70, and MPB80 of Mycobacterium bovis BCG. Infect Immun 52: 293-302.

5. Goraya JS, Virdi VS, 2001. Treatment of Calmette-Guerin bacillus adenitis: a metaanalysis. Pediatr Infect Dis J20: 632-634.

6. American Thoracic Society, CDC, Infectious Diseases Society of America, 2003. Treatment of tuberculosis. MMWR Recomm Rep 52: 1-77.
7. Siddiqi S, Rusch-Gerdes S, 2006. MGIT Procedure Manual for Bactec MGIT 960 TB System. Geneva: Switzerland: Foundation for Innovative new Diagnostics.

8. Brent AJ, Mugo D, Musyimi R, Mutiso A, Morpeth S, Levin M, Scott JA, 2011. Performance of the MGIT TBc identification test and meta-analysis of MPT64 assays for identification of the Mycobacterium tuberculosis complex in liquid culture. J Clin Microbiol 49: 4343-4346.

9. Yin X, Zheng L, Lin L, Hu Y, Zheng F, Hu Y, Wang Q, 2013. Commercial MPT64-based tests for rapid identification of Mycobacterium tuberculosis complex: a meta-analysis. J Infect 67: 369-377.

10. Warren RM et al., 2006. Differentiation of Mycobacterium tuberculosis complex by PCR amplification of genomic regions of difference. Int J Tuberc Lung Dis 10: 818-822.

11. Tsukamura M, Mizuno S, Toyama H, 1985. Taxonomic studies on the Mycobacterium tuberculosis series. Microbiol Immunol 29: 285-299.

12. Kasai H, Ezaki T, Harayama S, 2000. Differentiation of phylogenetically related slowly growing mycobacteria by their gyrB sequences. J Clin microbiol 38: 301-308.

13. Chaudhuri $A D, 2017$. Recent changes in technical and operational guidelines for tuberculosis control programme in India - 2016: a paradigm shift in tuberculosis control. J Assoc Chest Phys 5: 1-9.

14. Indumathi CK, Kowtal PM, Poornima RN, Lewin S, 2014. Clinical profile and outcome of clinical BCG disease in infants. Indian Pediatr 51: 730-732.

15. Cernuschi T, Malvolti S, Nickels E, Friede M, 2018. Bacillus Calmette-Guérin (BCG) vaccine: a global assessment of demand and supply balance. Vaccine 36: 498-506. 